\title{
Employees' Perception of Organizational Climate and Its Implications for Organizational Effectiveness in Amhara National Regional State, Ethiopia
}

\author{
Yitbarek Takele $^{1}$ and Mesfin Raji Kiltu ${ }^{2}$
}

\begin{abstract}
The growing significance placed on understanding of employees and their behaviour within the organization has produced a great deal of interest in investigating employees perceptions of climate within the organization especially, at times of significant change is taking place. The study aimed at determining the level of Organizational Climate (OC) as perceived by the civil servants and find out whether there is meaningful relationship between OC and Organizational effectiveness (OE). The research followed a quantitative approach and descriptive and causal designs while the data was analyzed using parametric statistical tools such as multiple linear regressions, Pearson's Product-moment Correlation Coefficients, Independent-samples T- test, and One-way ANOVA T-test. Overall, the findings are: the independent aggregate variables; Human Relations Values (HRV) and Open Systems Values (OSV) positively and significantly predicted the dependent variable (OE) and $32.5 \%$ of the variation in OE is explained by HRV and OSV combined. The research results will have implications to policy makers and future researches in that it might contribute for promoting a fundamental improvement in efficiency and performance of organizations as well as job satisfaction of employees.
\end{abstract}

Key Words: Organizational climate, Organizational effectiveness, Competing Values, Model, Amhara National Regional State, Ethiopia

DOI: http://dx.doi.org/10.4314/ejbe.v4i1.4

${ }^{1}$ Yitbarek Takele (PhD), Assistant Professor of Management, College of Business and Economics, Addis Ababa University, Ethiopia, Email: yitbarekt429@yahoo.com

${ }^{2}$ Mesfin Raji Kiltu, BSc and EMBA, Amhara Bureau of Labour and Social Affairs. Email: mesfinraji@gmail.com 
Implications of Employees' Perception of Organizational Climate

\section{Introduction}

The growing significance placed on understanding of employees and their behaviour within the organization has produced a great deal of interest in investigating employees perceptions of climate within the organization (Riggle 2007) especially, at times of significant change is taking place. Almost in all countries of the world including Ethiopia, people spend quite a bit of their time in organizations that could be public, business, or religious. From human behaviour perspective, we know that people have their own perceptions, feelings and attitudes towards the organisation structures and styles of management, their duties and responsibilities, and the conditions under which they are working. In fact, what is "perception" all about? How does it impart to factors of individual and group behaviours and influence organizational effectiveness? These are the keenly awaiting questions from the nomenclature of management theories.

According to Robbins (2004), perception can be defined as 'a process by which individuals organise and interpret their sensory impressions in order to give meaning to their environment' based on their perception of what reality is, not on reality itself; the world as it is perceived is the world that is behaviourally important. Of course, perception affects our working relationships in many ways relating to the factors of individual, group or organisational behaviour. Employee perceptions of OC and work experiences have become one of the relatively well researched aspects of management though the findings happen to be different in different contexts such as cultural differences, economic development, education, etc (Cooil et al. 2009). 
Implications of Employees' Perception of Organizational Climate

According to Schneider and Snyder (1975) each individual perceives his organization in any number of ways, depending upon the context and the set of information available about the organization. In this regard, OC is the employees' perception of how one feels to work in the unit, and includes specific aspects of the environment that directly affect people's ability to get the job done (Mullins 2005). This difference in perception is explained by Doherty and Horne (2002) as it might be because of missing, or misconstruing, some of the stimuli since a person's perception is the way they select, organize and interpret stimuli. Therefore, just as the perceptions of the individual are at the centre of any behavioural intervention in OC, so are the perceptions of the characteristics of the organization, by the members of the organization, central to the diagnosis of organization's problems and dysfunctions. This in turn will lead us to contemplating and understanding of how the OC, as perceived by employees in public sectors, is antecedent of the organization's effectiveness. Managers in public sectors so far have concentrated on accountability and high performance and have sought to restructure bureaucratic agencies, redefine organizational missions, streamline organizational processes, and decentralize decision-makings. Two of the more significant shifts in the $21^{\text {st }}$ century have been the increased attention to the delivery of public services on the one hand, and greater decentralization of responsibility for these services on the other (Ahmad et al. 2005). These facets of the service delivery have brought about much attention in public sectors to be more accountable and meet the expectations of the citizens.

Professional discharge of duties entails application of expertise and judgement and to large extent the motivation and direction of others and a positive contribution to the achievement of the objectives of the organization (Wachira 2011). Public discourse and public participation are important- particularly 
Implications of Employees' Perception of Organizational Climate

participation that involves citizens in making decisions about public services. Without an adequate understanding of OC or culture, many well-intended leaders have led organizations to their demise since it is important to address the social context in organizations (Agard 2011: 611- 612). This emphasizes the need to establish relationship between employees' perception of OC and its implication for organizational effectiveness in a given context. This need to be done on the basis of best instruments available after proper adaptation to contexts (Lok and Crawford 1999), as bureaucratic and stable environment often resulted in a lower level of employee commitment (Brewer, 1994; Kratina, 1990; Wallach, 1983) and performance (Krausz et al., 1995; Trice and Beyer, 1993). OC surveys routinely show that for about 75 percent of working adults the most stressful aspect of their job is the immediate boss (J. Hogan et al. 2009). Another study by Vijayakumar (2007) implied that the role of management style in shaping work climate perceptions of employees is crucial. The author advocated the case for strengthening participatory management style for a better perception of OC.

This study tries to explore the concept of OC from the viewpoint of employees' sensation that resides in the prevailing psychological environment of public institutions and the relevancy for quality and change initiatives. The growing significance placed on understanding civil servants behaviour within the organization has produced a great deal of interest in investigating their perceptions of climate within the organization. One of the likely future directions of employee attitude research as recommended by Saari \& Judge (2004) is better understanding the interplay between the person and the situation and the various internal and external factors that influence employee attitudes.

EJBE Vol. 4 No. $1 / 2014$

Page 119 
Implications of Employees' Perception of Organizational Climate

Therefore, the aim of this study was to analyse the effect of employees' perception of $\mathrm{OC}$ on organizational effectiveness in order to contribute (empirical) to the enrichment of the Amhara National Regional State (ANRS) civil service policy directed at improving organizational performance.

\section{Theoretical framework}

As part of their contribution on organizational behavior, Quinn and Rohrbaugh (1983) engaged in a series of studies to review the OC literature and have compiled a list of dimensions, which they termed as the "Competing Values Model" (CVM). Even though the model is likely rooted from Tom Burns mechanistic and organic type of classification of organizations in which the former is adapted to relatively stable conditions and the latter adapted to unstable conditions when new and unfamiliar problems continually arise (Pugh \& Hickson 1996), the CVM is a preferred way of portraying the organizational behavior in the context of organic type of organization that seeks adaptation to new environment. According to (K. S. Cameron \& Quinn, 1999; Quinn, 1988), the CVM is named as one of the fifty most important models in the history of business. It has since extended as a framework that makes sense of high performance with regards to numerous topics in the social sciences and organization studies. The CVM is studied and tested in organizations for more than twenty-five years by a group of thought leaders from leading business schools and corporations. It is still the topic of many books $\&$ research papers in the study of most aspects of organizations.

Though CVM suggests four main quadrants, each associated with different managerial ideologies encapsulating the means by which outcomes may be achieved (Cooil et al. 2009), two of its domains were used in this study as a 
Implications of Employees' Perception of Organizational Climate

theoretical framework: OSV \& HRV. While OSV as a frame of reference encompasses values such as flexibility, external organizational orientation, adaptability, the capacity to change, uniqueness and an orientation towards customers and the HRV includes internal focus, cohesion, morale, trust and belongingness (Cameron and Quinn 1999).

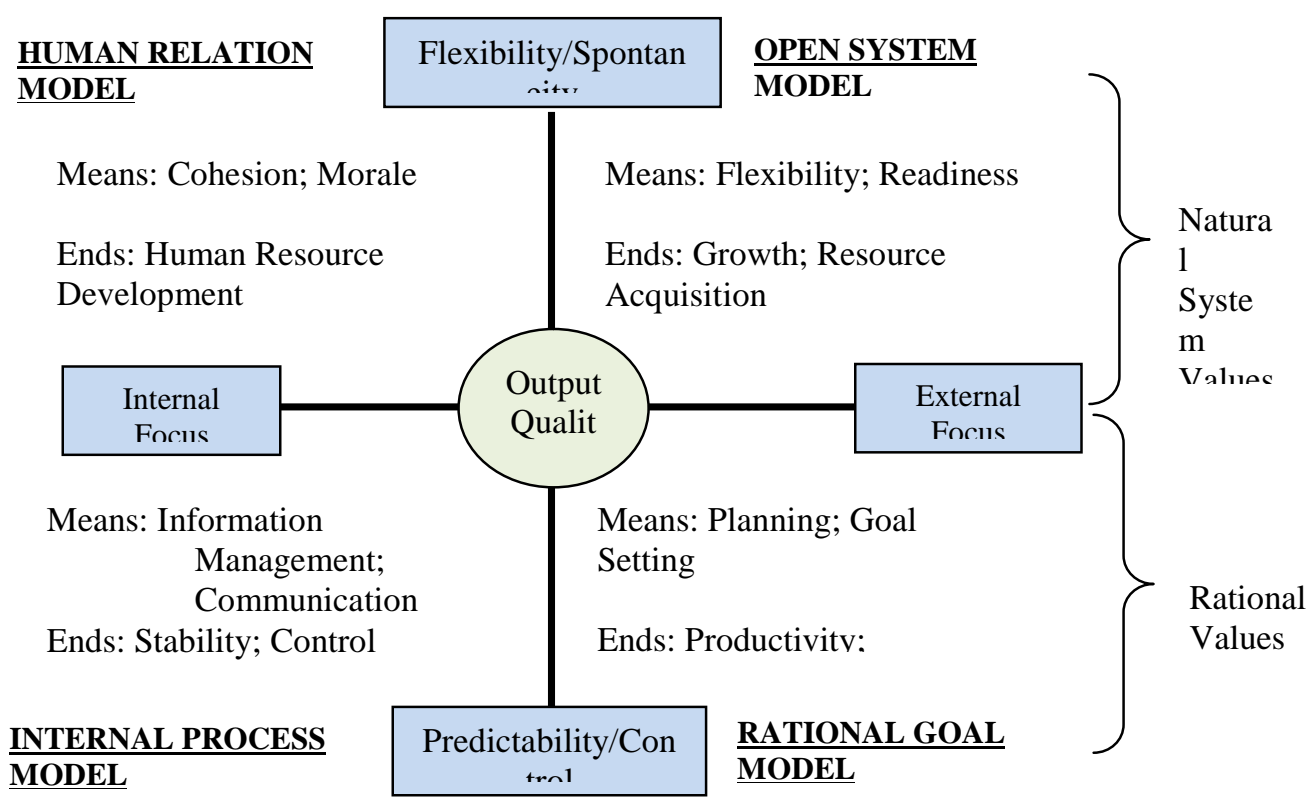

Source: Adapted from Quinn \& Rohrbaugh (1983: 369)

Fig-1: The Competing Values Framework

A major strength of this model is its derivation from four orientations to the study of organizational effectiveness, reflecting long traditions in work and organizational psychology (Patterson et al., 2004). Patterson and his colleagues thus, went on differentiating the two approaches from the other two in that the open systems approach emphasizes the interaction and adaptation of the 
Implications of Employees' Perception of Organizational Climate

organization in its environment, with managers seeking resources and innovating in response to environment. The human relations approach reflects the tradition derived from socio technical and human relations schools emphasizing the well-being, growth and commitment of the community of workers within the organization.

One of the main advantages of the CVM is the fact that it derives its approach from long standing theories in management and organizational psychology (Cooil et al. 2009) and it clarifies leadership roles and expectations and the clarification minimizes ambiguity and avoids interpersonal conflicts within teams(Zafft et al. 2009). The emergence of the pairs of competing values; flexibility versus stability, and internal versus external reflects a basic dilemma of organizational life and those organizations that are able to best balance integration and differentiation are the most effective systems. From which side we perceive, participants have unique feelings, likes and dislikes, and require consideration, appropriate information, and stability in their workplace (Quinn and Rohrbaugh 1983). Therefore, according to Quinn \& Rohrbaugh (1983), parallels among the models are important. The human relations and open system models share an emphasis upon flexibility whereas; the rational goal and internal process models are rooted in a value on control. Patterson et al. (2005) suggested that climate perceptions are associated with a variety of important outcomes at the individual, group, and organizational levels. They cited the various researches to support their argument on leader behaviour (Rousseau, 1988; Rentsch, 1990), turnover intentions (Rousseau, 1988; Rentsch, 1990), job satisfaction (Mathieu, Hoffman, and Farr, 1993; James and Tetrick, 1986; James and Jones, 1980), individual job performance (Brown and Leigh, 1996; Pritchard and Karasick, 1973), and organizational performance (Lawler et al., 1974; Patterson et al., 2004). Furthermore, as cited in (Imran et 
Implications of Employees' Perception of Organizational Climate

al. 2010), organizational commitment and job satisfaction were also found to be closely related (Glisson and James, 2002).

\section{The Research Model}

The study aimed at determining the nature and strength of perception of OC in relation to the human relations (internal focus) and open systems (external focus) of flexible organizational orientation of $\mathrm{OC}$ as perceived by the civil servants of the ANRS by assessing the demographic, group and organizational variations, and determine the implications to organizational effectiveness. The very reason for focusing on NSV than RV is as cited in (Schein 2004: p 172) Hawthorne studies (Roethlisberger and Dickson, 1939; Homans, 1950) had born the new series of "social" assumptions, postulating that employees motivated by the need to relate well to their peer and membership groups and that such motivation often overrides economic self-interest. This argument is against the many economic theories assumption that human beings are rational and employ utility maximization based on cost and benefit expectations as a way of understanding human behaviour (Bhattacherjee 2012). According to Bhattacherjee, political science theories assume that people are more political than rational, and try to position themselves in their professional or personal environment in a way that maximizes their power and control over others. This was also previously supported by (Ajzen 1991) in his theory of planned behaviour postulating that behaviours are based on one's intention regarding that behaviour, which in turn is a function of the person's attitude toward the behaviour, subjective norm regarding that behaviour, and perception of control over that behaviour. Therefore, for the reasons stated above, this research focused on Natural System Values than Rational values.

EJBE Vol. 4 No. $1 / 2014$

Page 123 


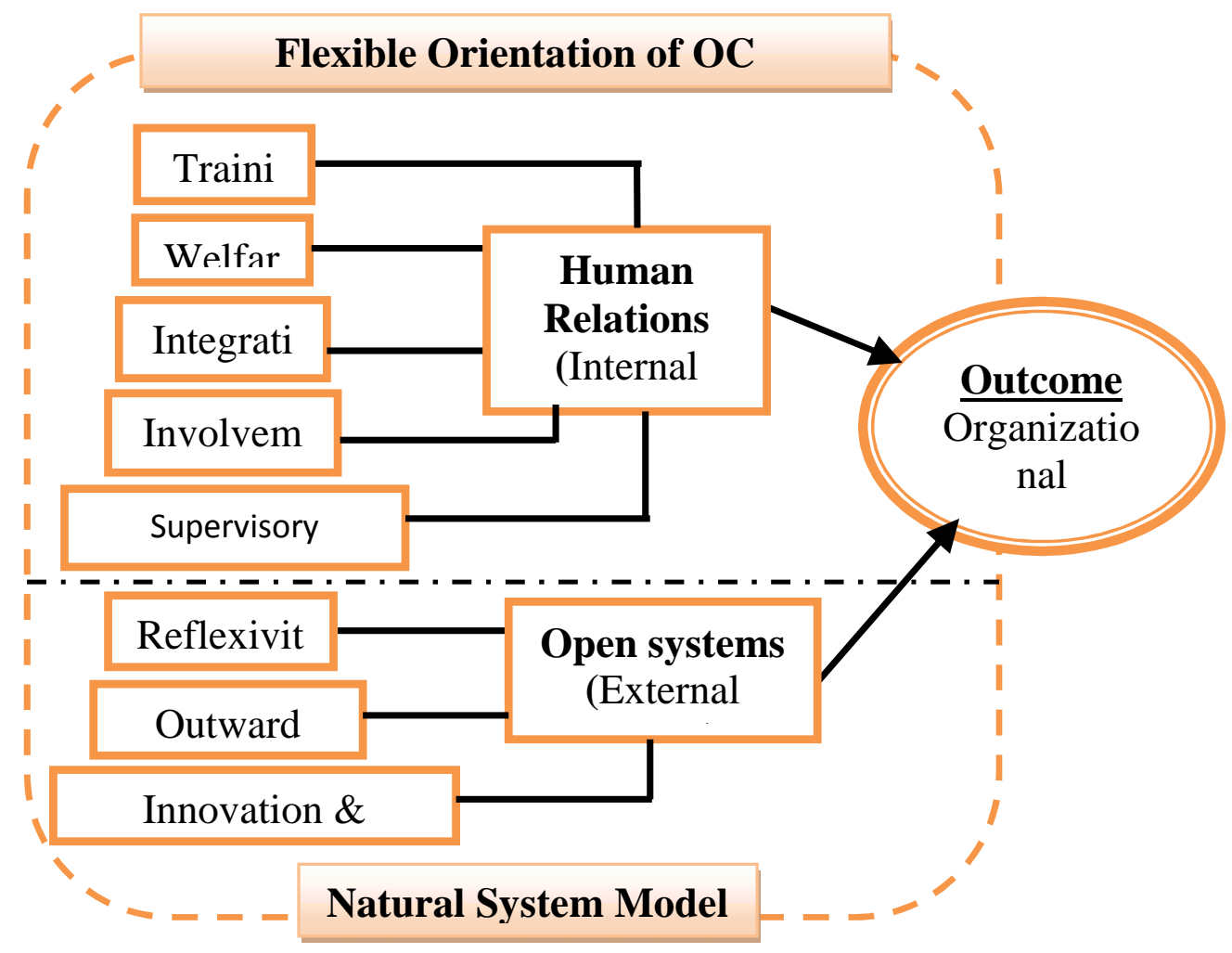

Fig-2: Research model adapted from Natural System Model of CVM

\section{Research hypotheses}

In the research, the following eight hypotheses were tested:

1) The aggregated HRV \& OSV of OC do not predict the organizational effectiveness that is measured by employees' organizational commitment.

2) The dimensions of OC (Integration, Involvement, Supervisory Support, Training, and Welfare) do not positively relate with HRV of flexible orientation. 
Implications of Employees' Perception of Organizational Climate

3) The dimensions of OC (Innovation \& flexibility, Reflexivity, and Outward focus) do not positively relate with OSV of flexible orientation.

4) There is no significant difference between employees' perception of OC in terms of employees' gender in HRV, OSV and OE across public sectors.

5) There is no significant difference between employees' perception of OC in terms of employees' tenure in HRV, OSV and OE across public sectors.

6) There is no significant difference between employees' perception of OC in terms of education level in HRV, OSV and OE across public sectors.

7) There is no significant difference between employees' perception of OC in terms of type of organization in HRV, OSV and OE across public sectors.

8) There is no significant difference between employees' perception of OC in terms of work processes in HRV, OSV and OE across public sectors.

\section{Research Approach and Methodology}

A quantitative research approach with descriptive and causal designs was applied along with a cross-sectional field survey method that made use of a self-administered questionnaire adopted to collect data such as numeric scores and biographic metrics. The sources of the primary data were all types of individual government civil servants excluding top ranked political appointees in the selected sampled public sectors. 
Implications of Employees' Perception of Organizational Climate

Sampling method and procedure

The study followed a stratified random sampling given the heterogeneous nature of the organizations included in the study. Moreover, taking the viability and representativeness of the sample, and resource constraints into consideration, $10 \%$ of the organizations were taken from each stratum as a representative sample frame. The stratification is done taking into account their difference in performance rankings in Civil Service Reform Program (CSRP) implementation, formally evaluated by the pertinent responsible body. The thirty-six organizations were classified based on the normal distribution curve of their rankings which shows a mean of $62.21 \%$ and standard deviation measure of $8.08 \%$. Assuming that the data is almost normally distributed, after transforming the data into standardized form and adjusting the Z-scores cut-off point between $+/-1.14$ and $+/-1.79$ points, 25 organizations $(69.44 \%)$ fall within average range of performers, 5 organizations (13.89\%) fall within the left $97.22 \%$ tail of data distribution, another 5 organizations (13.89\%) fall within the right $97.22 \%$ tail of data distribution, and only one organization falls outside the right extreme end of the $97.22 \%$ tail of the data distribution curve. Fig-3 shows the normal distribution curve of the sampling distribution of organizations. 
Implications of Employees' Perception of Organizational Climate

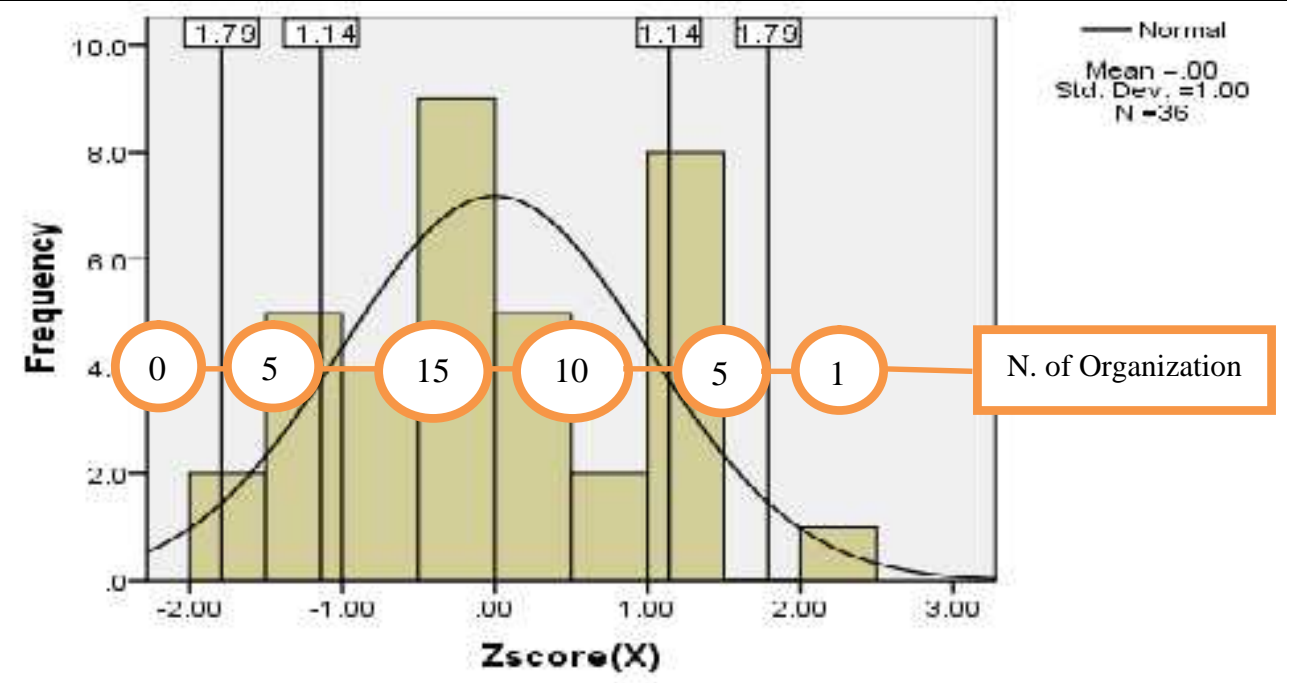

Fig-3: Sampling frame distribution based on performance of organizations

Hence, a sample size of 339 (45\%) individual respondents which were drawn from all work processes in random sampling out of the total 753 employees of the six organizations proportionately was determined using a formula for Sampling (Renckly 2002: 25).

\section{Method of data collection}

The study adopted the standardised, structured and validated OC measurement developed by Patterson et al (2005) based on competing values model (CVM). Only the flexible orientation values of HRV and OSV were tapped from the measurement. OE is measured indirectly using organizational commitment measures developed by (Speier \& Venkatesh 2002), which is adapted from (O'Reilly \& Chatman 1986). Organizational commitment has been identified as a useful measure of OE explaining the work-related behaviour of employees in organizations"(Iqbal 2007; Steers 1977) and the scales believed to measure the three basic components of organizational commitment: identification, involvement, and loyalty. Overall, the 8 dimensions of the OC measures 
Implications of Employees' Perception of Organizational Climate

comprised of 37 Likert-type items having a proven reliability test of 0.6 and above, the minimum score suggested by Hair et al.(1998) and Vijayakumar ( 2007) were retained from HRV and OSV of the CVM. For organizational commitment measure, three Likert-type items, which previous researchers, Speier \& Venkatesh (2002) have found with acceptable reliabilities of ( $\alpha=$ $0.75-0.83$ ) were used. The response formats for both types of measures used a 5 point, Likert-type scale from 1- Strongly Disagree to 5- Strongly Agree.

\section{Method of data analysis}

The collected data were statistically analysed by means of the Statistical Package for Social Sciences (SPSS Version 16) and the data analysis involved both descriptive and inferential statistics. Therefore, the collected data were analyzed using parametric statistical tools such as multiple linear regressions, Pearson's Product-moment Correlation Coefficients, Independent-samples Ttest, and One-way ANOVA to test the hypotheses.

\section{Variables of the study}

The organizational commitment measuring the $\mathrm{OE}$ was considered as dependent variable. The sub-scales of the HRV domain (Training, Welfare, Integration, Involvement, and Supervisory support) and OSV domain (Reflexivity, Outward focus and Innovation \& Flexibility) of OC were considered as first-order independent variables. The aggregated domains of HRV and OSV were considered as the second-order independent variables. 
Implications of Employees' Perception of Organizational Climate

\section{Results and Discussion}

\section{Validity and reliability analysis}

The construct validity of the instrument used was analysed using Principal Component Analysis, which is less complex than Factor Analysis and shares enormous similarity with Discriminant Analysis, a measure well known to capture construct validity (Field 2009). Since the Communality is a measure of the proportion of variance explained by the extracted factors, Stevens (2002) as mentioned in Field (2009: 644) recommends a loading value greater than 0.364 for a sample size of 200 with an alpha equal to .01 (two-tailed) and greater than 0.298 for a sample size of 300 or more. On the other hand, Hair et al. (1998) suggested that a cut-off loading value of 0.50 be adopted if the number of variables analyzed are many. In view of this, this study used a 0.5 cut-off loading value, given the large number of variables it used. Accordingly, a principal component analysis conducted on all first \& second order variables to examine their proposed single-factor structure (using "Eigenvalue $>1$ " rule) showed the total variance of HRV $=65.218 \%, \mathrm{OSV}=65.717 \%$, Integration $=$ $75.140 \%$, Involvement $=56.517 \%$, Supervisory Support $=69.697 \%$, Training $=62.106 \%$, Welfare $=77.705 \%$, Innovation \& Flexibility $=61.732 \%$, Outward Focus $=66.835 \%$, Reflexivity $=65.389 \%$, and $\mathrm{OE}=79.181 \%$. Item loading for the Single Factor in the component matrix for all variables that contain the item loading showed that all of the items are loaded strongly onto their respective components with all the items having loadings above the 0.5 cut-off loading value. Besides, the KMO value ranged from .500 to .914 , which is on or above the cut-off point of 0.5 recommended by Kaiser (1974). The KMO values closer to 1 indicate the relatively compact patterns of the correlations indicating factors that are distinct and reliable. 
Implications of Employees' Perception of Organizational Climate

The split-half method is used to test the internal consistency levels of the measuring instrument (Field 2009; Nunnally 1978). In so doing, the Cronbach alpha value of all variables were calculated and the results were the following: $\mathrm{HRV}$ scale $=0.900, \mathrm{OSV}$ scale $=0.835$, Integration scale $=0.667$, Involvement scale $=0.615$, Supervisory Support scale $=0.891$, training scale $=0.691$, Welfare scale $=0.856$, Innovation \& Flexibility scale was 0.875 , Outward Focus scale $=0.751$, Reflexivity scale $=0.735$, and $\mathrm{OE}$ scale $=0.868$ which is either not substantially low or above the 0.70 criterion of Nunnally (1978). Therefore the reliability of all measurement scales used in this study can be considered acceptable (Kline 1999 as cited in Field 2009: 675).

\section{Descriptive statistics}

As key measures of descriptive statistics, mean, standard deviation, skewness and kurtosis were used in the study (Field 2009; Veaux et al. 2008; Collis \& Hussey 2003). Use of standard deviation is important as it indicates the dispersion of the data (De Veaux et al. 2008). Skewness and kurtosis are also essential to know whether the distribution is normal where a positive skewness value indicates too many low scores in the distribution and negative values indicate a build-up of high scores. Positive values of kurtosis indicate a pointy and heavy-tailed distribution, whereas negative values indicate a flat and lighttailed distribution (Field 2009).

Skewness and kurtosis are converted to $z$-scores using the following formulas

$\mathrm{Z}_{\text {skewness }}=\frac{S-0}{S E_{\text {skewness }}} \quad \mathrm{Z}_{\text {kurtosis }}=\frac{K-0}{S E_{\text {kurtosis }}}$

An absolute value of $z$-scores greater than 1.96 is significant at $p<.05$, above 2.58 is significant at $p<.01$ and absolute values above 3.29 are significant at $p$ 
Implications of Employees' Perception of Organizational Climate

$<.001$. Large samples will give rise to small standard errors and when sample sizes are big, the 1.96 criterion should be increased to the 2.58 (Field 2009: 139). Therefore, through Case wise diagnostics for the sample size of 260 we were able to verify that there is no case that falls outside the limit of $+/-2.58$ indicating that the sample is normally distributed at $1 \%$ significance level.

The descriptive statistics result on sub-scales of OC shows that the Integration variable has a mean of 3.34 , a median of 3.50 , and a standard deviation of 0.79 . It is a negatively skewed $\left(Z_{s=-0.35}\right)$ and has negative kurtosis $\left(Z_{k=-0.06)}\right.$. The Involvement variable has a mean of 3.15, a median of 3.17, and a standard deviation of 0.73 . It is a negatively skewed distribution $\left(\mathrm{Z}_{\mathrm{s}=-0.01)}\right.$ and with

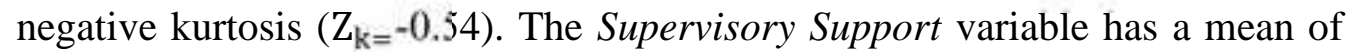
3.15 , a median of 3.20 , and a standard deviation of 1.02 . It is a negatively skewed distribution $\left(Z_{s=-0.21)}\right.$ and with negative kurtosis $\left(Z_{k=-0.77)}\right.$. The Training variable has a mean of 2.91 , a median of 2.67 , and a standard deviation of 0.97. Its skewness is almost zero and with negative kurtosis $\left(\mathrm{Z}_{\mathrm{k}=-}\right.$ 0.57). Innovation \& Flexibility variable has a mean of 3.00, a median of 3.00 , and a standard deviation of 0.84 . It is a negatively skewed distribution $\left(\mathrm{Z}_{\mathrm{s}=-}\right.$

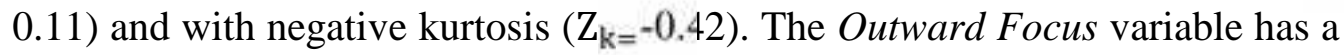
mean of 3.68 , a median of 4.00 , and a standard deviation of 0.70 . It is a

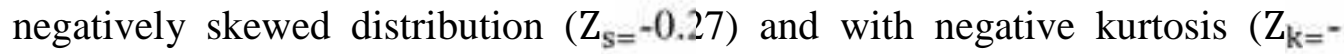
0.39). The Reflexivity variable has a mean of 3.39 , a median of 3.33 , and a standard deviation of 0.84 . It is a negatively skewed distribution $\left(\mathrm{Z}_{\mathrm{s}=-}=0.48\right)$ and with positive kurtosis $\left(Z_{k=0.08)}\right.$. The aggregated second-order $O C$ variables show that $H R V$ has a mean of 3.11 , a median of 3.09 , and a standard deviation of 0.72 . It is a positively skewed distribution $\left(\mathrm{Z}_{\mathrm{s}=0.05)}\right.$ and with negative kurtosis $\left(\mathrm{Z}_{\mathrm{k}=-0.60)}\right.$. OSV has a mean of 3.30, a median of 3.31, and a standard 
Implications of Employees' Perception of Organizational Climate

deviation of 0.66 . It is a negatively skewed distribution $\left(\mathrm{Z}_{\mathrm{s}=-0.24)}\right.$ and with

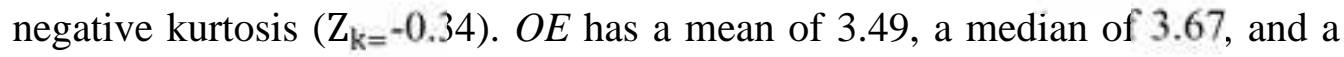
standard deviation of 1.06. It is a negatively skewed distribution $\left(Z_{s=}=0.49\right)$ and

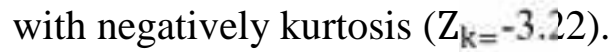

\section{Hypothesis testing}

Null hypothesis 1: The aggregated HRV \& OSV of OC do not predict $O E$

Multiple linear regression was run one time to test the effect of HRV and OSV on $\mathrm{OE}$ using the following regression model.

$Y=\alpha_{0}+b_{1} X_{1}+b_{2} X_{2}+\epsilon$

\section{Where:}

$\mathrm{Y}=$ Organizational Effectiveness $(\mathrm{OE}), \mathrm{X}_{\mathrm{i}}=$ the independent Variables in which; $X_{1}=$ Human Relations Values (HRV), $X_{2}=$ Open System values $(\mathrm{OSV}), \alpha_{0}=$ the $\mathrm{y}$-intercept, $\mathrm{b}_{\mathrm{i}, \mathrm{i}=1-2}=$ regression coefficients of the two factors in explaining $\mathrm{Y}$, and $\epsilon=$ the Error term.

Hence, $H R V$ and $O S V$ significantly predicted $O E(\boldsymbol{\beta}=\mathbf{0 . 2 7 2}, \mathbf{p}<\mathbf{0 . 0 5}$ and $\boldsymbol{\beta}=$ $\mathbf{0 . 3 3 8}, \mathrm{p}<\mathbf{0 . 0 5}$, respectively) with no problem of multicollinearity and autocorrelation. The strength of the relationship as indicated by the adjusted $R$ Square value accounts 0.319 and is considered as low positive correlation (Collis \& Hussey 2003) indicating little or no multicolliniarity problem. Of course, autocorrelation is a problem in time-series studies but not in crosssectional (Manly 2009: 191). Nonetheless, the Durbin-Watson test with a value of 1.974 proves the above claim. 


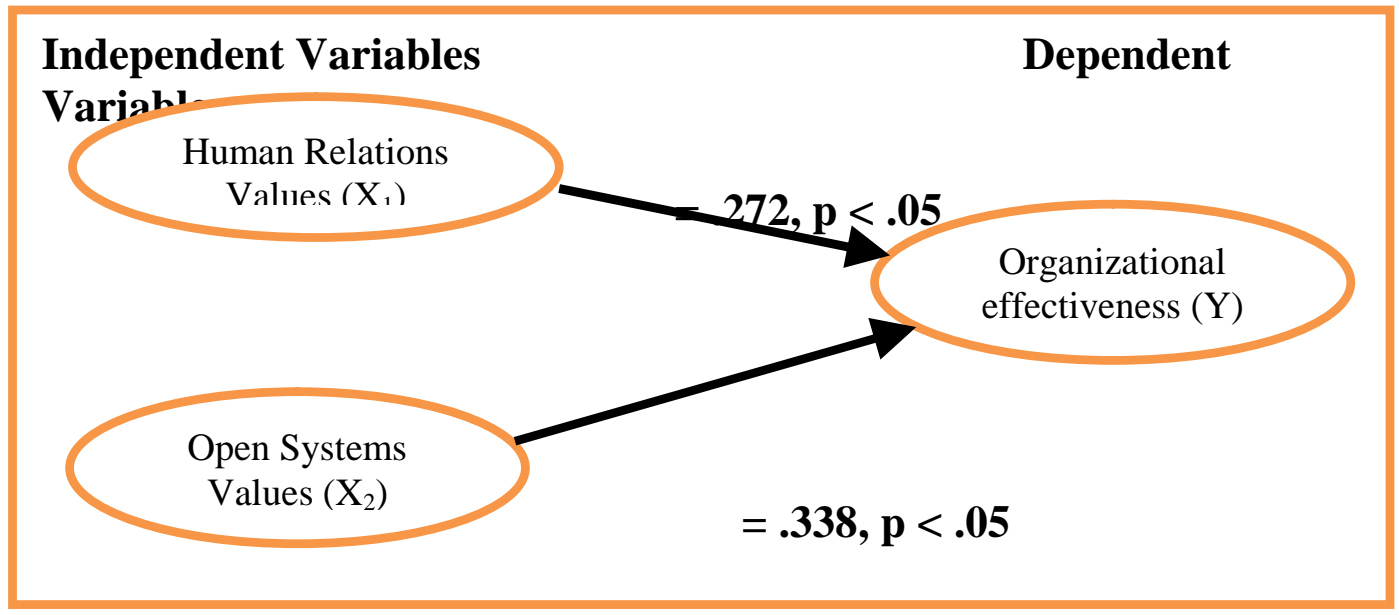

Fig-4: Relationship between HRV and OSV on OE

The above regression result is re-written as follows:

$O E=1.021+0.401(H R V)+0.412(O S V)$

Most importantly, in the model summary, $R$ square of .325 indicates $32.5 \%$ of the variation in $O E$ is explained by $H R V \& O S V$ combined. The remaining $67.5 \%$ variation in $O E$ is explained by factors not included in the model, essentially, by the other two rational values in the quadrants of CVM; rational goal values $(R G V) \&$ internal process values (IPV). $R G V \& I P V$ contribute almost $65 \%$ of the variation on the half of the CVM. In a summary, the multiple linear regression test undertaken above proved that the null hypothesis 1 is rejected.

Null hypothesis 2: The dimensions of $O C$ (Integration, Involvement, Supervisory Support, Training, and Welfare) are not positively related with $H R V$ of flexible orientation. 
Implications of Employees' Perception of Organizational Climate

Pearson's product moment correlation coefficient $(r)$ is used to determine the strength of association between two variables(Collis \& Hussey 2003). Therefore, the result indicated that Involvement, Supervisory Support, Training and Welfare have shown high positive correlation with $H R V(r=\mathbf{. 7 3 6}, \mathbf{. 8 6 8}$, . $\mathbf{7 6 6}$, and .848 at $\mathbf{p}<\mathbf{0 . 0 1}$ respectively). In contrast, Integration has shown medium positive correlation with $H R V(\boldsymbol{r}=\mathbf{. 6 2 2})$.

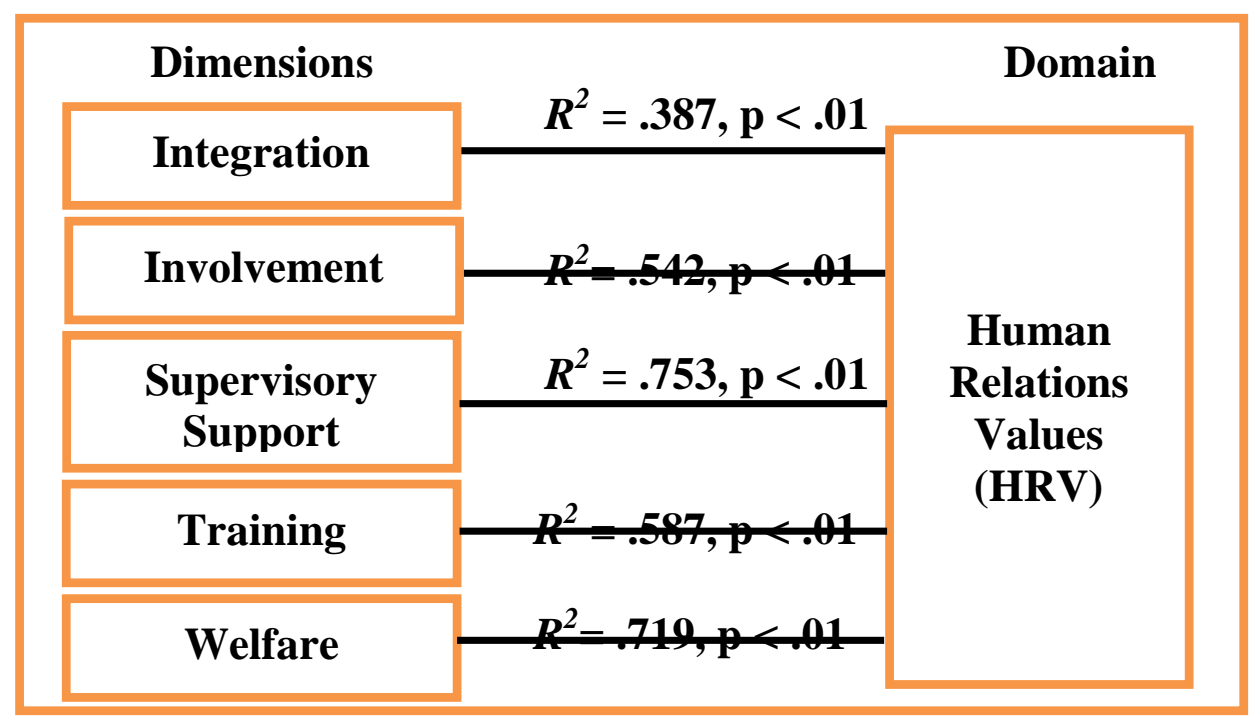

\section{Fig-5: Relationships of dimensions of OC with HRV}

The coefficient of determination, $\boldsymbol{R}^{2}$ that determines the amount of variability in one variable that is shared by the other (Field 2009), shows that Integration, Involvement, Supervisory Support, Training and Welfare account for 38.7\%, $54.2 \%, 75.3 \%, 58.7 \%$, and $71.9 \%$ of the variation in $H R V$ respectively. The sub-scales were regressed against the dependent variable $H R V$, and their combined effect found to account $95.3 \%$ of the variability in HRV. In a summary, Pearson's product-moment correlation coefficient analysis carried out proved that the null hypothesis 2 is rejected.

EJBE Vol. 4 No. $1 / 2014$ 
Implications of Employees' Perception of Organizational Climate Null hypothesis 3: The dimensions of OC (Innovation \& flexibility, Reflexivity, and Outward focus) do not positively related with OSV of flexible orientation.

Pearson's product-moment correlation coefficient $(r)$ was used to determine the strength of association between two variables(Collis \& Hussey 2003). Therefore, the result indicated that Innovation \& Flexibility has shown very high positive correlation with $O S V(r=.987 ; \mathbf{p}<\mathbf{0 . 0 1})$. In contrast, Reflexivity has demonstrated medium positive correlation with $\operatorname{OSV}(\boldsymbol{r}=\mathbf{. 6 7 7}$ at $\mathbf{p}<\mathbf{0 . 0 1})$ and Outward Focus has demonstrated low positive correlation with $\operatorname{OSV}(\boldsymbol{r}=$ .367 at p < 0.01)(Collis \& Hussey 2003; Patterson et al. 2005).

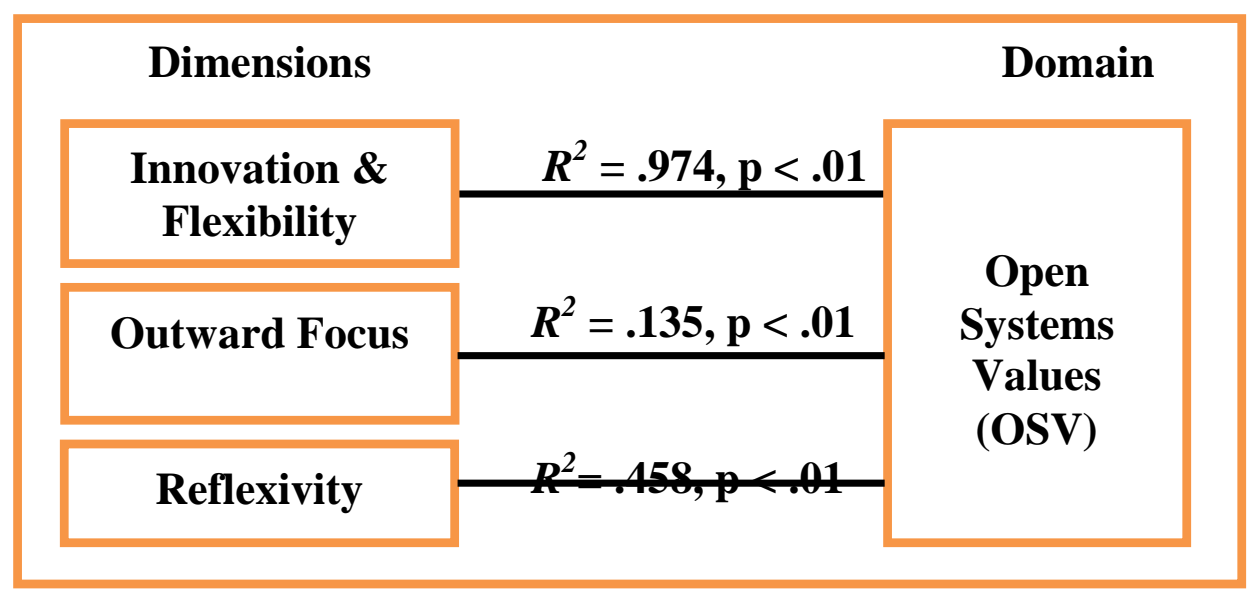

\section{Fig-6: Relationships of dimensions of OC with OSV}

The coefficient of determination, $\boldsymbol{R}^{\mathbf{2}}$ shows that Innovation \& Flexibility, Outward Focus, and Reflexivity account for 97.4\%, 13.5\%, and 45.8\% of the variation in $O S V$ respectively. The sub-scales were regressed against the dependent variable $O S V$, and their combined effect found to exclude the Outward focus and Flexibility from the model and the remaining factor account 97.4\% of the variability in HRV. In a summary, Pearson's product-moment 
Implications of Employees' Perception of Organizational Climate

correlation coefficient analysis carried out proved that the null hypothesis 3 is rejected.

Null hypothesis 4: There is no significant difference between employees' perception of $O C$ in terms of employees' Gender in HRV, OSV and OE across public sectors.

Independent Samples t-test was used to establish whether two means collected from independent samples differ significantly (Field 2009). Therefore, the result indicated that on average, Males experienced greater positive perception to $O E(M=3.53, S E=0.09)$ than Females $(M=3.42, S E=0.10)$, greater positive perception to $H R V(M=3.06, S E=0.06)$ than Females $(M=3.05, S E$ $=0.07)$, and greater positive perception to $O S V(\boldsymbol{M}=\mathbf{3 . 0 4}, \boldsymbol{S E}=\mathbf{0 . 0 7})$ than Females $(\boldsymbol{M}=\mathbf{2 . 9 6}, \boldsymbol{S E}=\mathbf{0 . 0 9})$. The difference in perception in all cases was not significant $(\mathbf{t}(\mathbf{2 0 9 . 6})=.829, p>.05),(t)(258)=.151, p>.05)$ and $(\mathbf{t}(258)$ $=\mathbf{. 6 8 5}, p>\mathbf{. 0 5}$ ) than Females respectively. This will lead us to a conclusion that males and females do not differ in their perception of OC and on OE. In a summary, Independent-Samples t-test that was carried out proved that there is no ground for the null hypothesis 4 to be rejected for $\mathrm{OC}$ and $\mathrm{OE}$.

Null hypothesis 5: There is no significant difference between employees' perception of OC in terms of employees' Tenure in HRV, OSV and OE across public sectors.

One-way independent ANOVA test was used to test for differences between years of experiences of independent groups. Therefore, the result indicated that there was no significant difference in Tenure exhibited in a period of job possession in terms of employees perception of $\mathrm{OC}$ and organizational commitment; $O E, \boldsymbol{F}(\mathbf{4}, \mathbf{2 4 5})=\mathbf{1 . 1 5 1}, \boldsymbol{p}>.05, H R V, \boldsymbol{F}(\mathbf{4}, \mathbf{2 4 5})=.918, \boldsymbol{p}>.05$, 
Implications of Employees' Perception of Organizational Climate

and $O S V, \boldsymbol{F}(\mathbf{4}, \mathbf{2 4 5})=\mathbf{. 8 6 4}, \boldsymbol{p}>\mathbf{. 0 5}$. In a summary, One-way Independent ANOVA test that was carried out proved that there was no ground for the null hypothesis 5 to be rejected for OC factors of HRV \& OSV and OE.

Null hypothesis 6: There is no significant difference between employees' perception of $O C$ in terms of Education level in human relations, $O S V$ and $O E$ across public sectors.

One-way independent ANOVA test was used to test for differences between levels of education of independent groups. Therefore, the result indicated that there was no significant difference in Education level exhibited whether the employee is qualified or not in terms of employees' perception of OC and organizational commitment. $O E, \boldsymbol{F}(\mathbf{4}, \mathbf{2 4 7})=\mathbf{1 . 9 2}, \boldsymbol{p}>\mathbf{. 0 5}, H R V, \boldsymbol{F}(\mathbf{4}, \mathbf{2 4 7})=$ $\mathbf{3 6 9}, \boldsymbol{p}>\mathbf{. 0 5}$, and $O S V, \boldsymbol{F}(\mathbf{4}, \mathbf{2 4 7})=\mathbf{. 9 9 5}, \boldsymbol{p}>\mathbf{. 0 5}$. In a summary, One-way Independent ANOVA test that was carried out proved that there was no ground for the null hypothesis 6 to be rejected for OC factors of HRV \& OSV and OE.

Null hypothesis 7: There is no significant difference between employees' perception of $O C$ in terms of the type of organization in HRV, OSV and $O E$ across public sectors.

One-way independent ANOVA test and Independent-sample t-test were used to test for differences between the different sampled organizations. Therefore, the result indicated that there is significant difference in employees perception across Organizations in terms of $O E(\boldsymbol{F}(\mathbf{5}, \mathbf{2 5 4})=\mathbf{2 . 3 9 5}, \boldsymbol{p}<\mathbf{. 0 5})$ with a mean of; ORG-A = 3.84, ORG-B = 3.67, ORG-C = 3.53, ORG-D = 3.47, ORG-E = 3.28 , and ORG-F $=3.19$. Significant difference in employees perception across Organizations in terms of $\operatorname{OSV}(\boldsymbol{F}(\mathbf{5}, \mathbf{2 5 4})=\mathbf{3 . 6 7}, \boldsymbol{p}<. \mathbf{0 5})$ with a mean of 
Implications of Employees' Perception of Organizational Climate ORG-A $=3.39$, ORG-C $=3.09$, ORG-D $=2.99$, ORG-B $=2.93$, ORG-E $=$ 2.91 , and ORG-F $=2.65$ of the OC. On other hand, no significanct difference was observed in $H R V(\boldsymbol{F}(\mathbf{5}, \mathbf{2 5 4})=\mathbf{2 . 0 8 1}, \boldsymbol{p}>\mathbf{. 0 5})$ of employees perception across public organizations.

Table-1: Significance of difference for organization types

\begin{tabular}{|l|l|l|l|}
\hline \multirow{2}{*}{$\begin{array}{l}\text { Pair of groups for } \\
\text { Organizations }\end{array}$} & \multicolumn{2}{ll}{ t-values } & OEV \\
\hline ORG-E Vs ORG-B & -1.726 & .321 & -.114 \\
\hline ORG-E Vs ORG-D & -.778 & -1.421 & -.411 \\
\hline ORG-E Vs ORG-A & $-2.691 * *$ & -1.724 & $-3.021 * *$ \\
\hline ORG-E Vs ORG-C & -1.193 & -.121 & -1.041 \\
\hline ORG-E Vs ORG-F & .397 & 1.301 & 1.654 \\
\hline ORG-B Vs ORG-D & .814 & -1.581 & -.282 \\
\hline ORG-B Vs ORG-A & -.767 & -1.839 & $-2.617 *$ \\
\hline ORG-B Vs ORG-C & .589 & -.400 & -.853 \\
\hline ORG-B Vs ORG-F & $2.152 *$ & .889 & 1.733 \\
\hline ORG-D Vs ORG-A & -1.520 & -.134 & -1.930 \\
\hline ORG-D Vs ORG-C & -.249 & 1.212 & -.426 \\
\hline ORG-D Vs ORG-F & 1.126 & $2.542 *$ & 1.713 \\
\hline ORG-A Vs ORG-C & 1.419 & 1.479 & 1.664 \\
\hline ORG-A Vs ORG-F & $2.948^{*} *$ & $2.851 * *$ & $4.603 * * *$ \\
\hline ORG-C Vs ORG-F & 1.497 & 1.312 & $2.548^{*}$ \\
\hline
\end{tabular}

Notes: $* \mathrm{p}<=.05, * * \mathrm{p}<=.01$ and $* * * \mathrm{p}<=.001$

Table 1 above shows the result of Independent-sample t-test run for each grouping that $9(20 \%)$ out of 45 tests conducted revealed statistically significant differences in the perception of organizational climate. At least two significant differences exist in each factor. At least one significant difference exists for each pair of organization grouping. Organizational climate is, therefore, not uniform, but differential across various groups across organizations. In a summary, One-way Independent ANOVA test undertaken above proved that the null hypothesis 7 can be rejected for OC factor of OSV and OE. However, 
Implications of Employees' Perception of Organizational Climate

there is no ground for the null hypothesis 7 to be rejected for OC factor of HRV.

Null hypothesis 8: There is no significant difference between employees' perception of $O C$ in terms of work processes in HRV, OSV and OE across public sectors.

Independent Samples t-test was used to determine the difference and its significance by comparing the means of two work groups. Therefore, the result indicated that on average, employees in Core processes experienced greater positive perception to $O E(\boldsymbol{M}=\mathbf{3 . 5 6}, \boldsymbol{S E}=\mathbf{0 . 0 8})$ than employees in Support processes $(\boldsymbol{M}=\mathbf{3 . 3 9}, \boldsymbol{S E}=\mathbf{0 . 1 1})$. On other hand, on average, employees in Support processes experienced greater positive perception to $H R V(\boldsymbol{M}=\mathbf{3 . 0 8}$, $\boldsymbol{S E}=\mathbf{0 . 0 7})$ than employees in Core processes $(\boldsymbol{M}=\mathbf{3 . 0 4}, \boldsymbol{S} \boldsymbol{E}=\mathbf{0 . 0 6})$ and on average, employees in Support processes experienced almost equal positive perception to $O S V(\boldsymbol{M}=\mathbf{3 . 0 1}, \boldsymbol{S E}=\mathbf{0 . 0 9})$ with employees in Core processes ( $\boldsymbol{M}$ $=3.01, S E=0.07)$. However, the differences in $O E \& H R V$ were not significant $\mathbf{t}(\mathbf{2 5 8})=\mathbf{1 . 2 9 1}, \boldsymbol{p}>\mathbf{. 0 5}$ for $O E$; not significant $\mathbf{t}(\mathbf{2 5 8})=\mathbf{- . 3 8 1}, \boldsymbol{p}>$ .05 for $H R V$; and not significant $\mathbf{t}(\mathbf{2 5 8})=\mathbf{. 0 3 8}, \boldsymbol{p}>.05$ for $O S V$. In a summary, Independent-Samples t-test that was carried out proved that there is no ground for the null hypothesis 8 to be rejected for $\mathrm{OE}$ as well as for $\mathrm{OC}$ factors of HRV and OSV.

\section{Conclusion, Policy and Future Research Implications}

The research findings on Hypothesis 1 were consistent with prior theory and research in that $O C$ positively \& significantly influences on employees' perception of their work environment and impacts upon $O E$ (Quinn and 
Implications of Employees' Perception of Organizational Climate

Rohrbaugh 1983). Employees' psychological capital is positively related to their performance, satisfaction, and commitment and that a supportive climate is related to employees' satisfaction and commitment (Bakker \& Schaufeli 2008). Moreover, according to CVM, nearly $50 \%$ of the variation in OE should have to be explained by HRV \& OSV. However, in this study, 32.5\% of the variation was found to account by these two factors (Quinn and Rohrbaugh 1983). This indicates that in the natural system approach employees perceive that $\mathrm{OC}$ is important (exhibit high $H R V$ such as morale and cohesion, and high $O S V$ such as flexibility and readiness) and has an impact on their behavioral intentions to give more emphasis on human resource development and growth and resource acquisition. This, in turn, places $\mathrm{OC}$ as a leading indicator of organisational performance and as a key motivational factor for organizational change that contributes to $O E$ (Srivastav 2009; Lehman et al. 2002). The research question has been answered, in that the OE of public sectors in ANRS that is measured by employees' organizational commitment is highly predicted by the flexible orientation on internal and external values of OC.

The research findings on Hypotheses 2 and 3 were consistent with prior theory and research conducted on competing values framework that the climate strength of all or most climate dimensions within a CVM quadrant intercorrelate significantly as predictors of organizational outcomes such as organizational commitment (Iqbal 2007; Patterson et al. 2005). The results have shown that employees of public institutions do not only perceive the dimensions in the $H R V$ and $O S V$ domain of OC positively, but also their perception has strong effect on their behavioural intentions of $H R V$ such as morale, cohesion, trust and belongingness. This is true also for $O S V$ that encompasses values such as flexibility, external organizational orientation, adaptability, the capacity to change, uniqueness and an orientation towards 
Implications of Employees' Perception of Organizational Climate

customers (Reino \& Vadi 2010). The findings also reveal that in relative terms Supervisory support and Innovation \& Flexibility found to be the dominant factors under $H R V$ and $O S V$ domain of $\mathrm{OC}$ in influencing employees' perception respectively. Integration and Outward Focus are the least among the HRV and OSV domain of OC in influencing employees' perception respectively. Hence, the research questions have been answered, in that there is significant positive relationship between the dimensions of OC and both HRV (internal focus) and OSV (external focus) of flexible orientation.

The research findings on hypotheses 4 and 8 were consistent with the previous research that states males and females do not differ in their perception of OC (Idogho 2006). On the contrary, contradictory result obtained with regard to work processes with previously studied related topics that states there is variation in level of agreement both across teams within samples and within teams across particular dimensions (N. R. Anderson and M. A. West 1998; Srivastav 2009). However, it cannot arguably be ruled out that the organizational dynamism could have significant impact in today's work environment than long time ago to rely more on latest phenomena. Hence, the research questions have been answered, in that there is no significant difference between employees' perception of OC in terms of employees' gender and work processes in $H R V$, $O S V$ and $O E$ across public sectors.

The research findings on hypotheses 5, 6 and 7 were consistent with the previous research conducted on academic staff of universities in which no differences exist between young and older academic staff (Idogho 2006). In terms of education level, even though no previous research was found to contrast with, the findings proved that employees' background in educational qualification has nothing to do with employees' perception towards OC. In 
Implications of Employees' Perception of Organizational Climate

terms of differences in organization type, partial result obtained from the previous research conducted on academic staff of Universities that indicated the existence of significant difference among universities in their perception of OC (ibid) even if the units of analysis are aggregated by human relations or open systems values. The measuring instrument should demonstrate significant differences in employee perceptions across organizations if it is to be useful in discriminating between organizations (Patterson et al., 2005). Hence, the research questions have been addressed, in that there is no significant difference between employees' perception of OC in terms of employees' tenure and education level in $H R V, O S V$ and $O E$ across public sectors. On the other hand, there is significant difference between employees' perception of $O C$ in terms of organization type in $O E \& O S V$. However, there is no significant difference between employees' perception of OC in terms of organization type in $H R V$.

In view of the above conclusions, the following are suggested to be inputs to any policy formulations and reviews. As $32.5 \%$ of the variation in $\mathrm{OE}$ is explained by HRV \& OSV combined and the remainder of the variation by rational goal values (RGV) \& internal process values (IPV), ANRS shall focus on rational values to improve its Organizational climate. Second, the dimensions of OC that include Integration, Involvement, Supervisory Support, Training, and Welfare shall be used to promote human resource development. Finally, ANRS shall work on interventions that improve OSV flexible climate dimensions except outward focus (Innovation, Flexibility and Reflexivity) as they are proved to be good predictors of organizational outcomes. 
Implications of Employees' Perception of Organizational Climate

Finally, it is suggested that any future research on OC shall comprehensively address both dimensions of NSV and RV as the current study was limited to NSV and was able to explain only $32.5 \%$ of the variation in OC in ANRS. 
Implications of Employees' Perception of Organizational Climate

\section{References}

Agard, K.A., 2011. Leadership in Non-profit Organizations: A Reference Handbook, USA: SAGE Publications, Inc.

Ahmad, J. et al., 2005. Decentralization and Service Delivery. World Bank, (3603), pp.1-29.

Anderson, N.R. \& West, M.A., 1998. Measuring climate for work group innovation: development and validation of the team climate inventory. Journal of Organizational Behavior, 19(June 1996), pp.235-258.

Bakker, A.B. \& Schaufeli, W.B., 2008. Positive organizational behavior: Engaged employees in flourishing organizations. Journal of Organizational Behavior, 29, pp.147-154.

Cameron, K. S. \& Quinn, R.E., 1999. Diagnosing and Changing Organizational Culture. Based on the Competing Values Framework, Massachusetts: Addison - Wesley Longman.

Cameron, K.S \& Quinn, R.E., 1999. Diagnosing and changing organizational culture, Reading, MA.: Addison-Wesley Publishing Company, Inc.

Collis, J. \& Hussey, R., 2003. Business Research: A Practical Guide for Undergraduate and Postgraduate Students, Palgrave Macmillan (UK).

Cooil, B. et al., 2009. The Relationship of Employee Perceptions of Organizational Climate to Business-Unit Outcomes: An MPLS Approach. , (804), pp.1-49.

Doherty, T.L. \& Horne, T., 2002. Managing Public Services - Implementing Changes: A thoughtful approach to the practice of management,

Field, A., 2009. Discovering Statistics Using SPSS 3rd ed., SAGE Publications, Inc.

Hair, J.F. et al., 1998. Multivariate Data Analysis 5th ed., Upper Saddle River, NJ: Prentice Hall. 
Implications of Employees' Perception of Organizational Climate

Hogan, J., Hogan, R. \& Kaiser, R.B., 2009. Management Derailment: Personality Assessment and Mitigation. Working paper, pp.1-28.

Idogho, P.O., 2006. Academic Staff Perception of the Organizational Climate in Universities in Edo State, Nigeria. Journal of Social Science, 13(1), pp.71-78.

Imran, R. et al., 2010. Organizational climate as a predictor of innovative work behavior. African Journal of Business Management, 4(15), pp.3337-3343.

Iqbal, A., 2007. Organizational climate and employees' commitment: a study of the Pakistani knitwear industry. , pp.1-6.

Lehman, W.E.K., Greener, J.M. \& Simpson, D.D., 2002. Assessing organizational readiness for change. Journal of Substance Abuse Treatment, 22, p.200.

Linden, R.M., 1998. Workbook for seamless Government: A hands-on Guide to implementing Organizational Change, San Francisco, California: JosseyBass Inc.

Lok, P. \& Crawford, J., 1999. The relationship between commitment and organizational culture, subculture, leadership style and job satisfaction in organizational change and development. Leadership \& Organization Development Journal, 20(7), pp.365-373.

Manly, B.F.J., 2009. Statistics for Environmental Science and Management 2nd ed. R. Smith, ed., New York: Taylor \& Francis Group, LLC.

Mullins, L.J., 2005. Management and Organisational Behaviour 7th ed.,

O'Reilly, C. \& Chatman, J., 1986. Organizational commitment and psychological attachment:The effects of compliance, identification, and internalization on prosocial behavior. Journal of Applied Psychology, 71(3), pp.492-499. 
Implications of Employees' Perception of Organizational Climate

Patterson, M.G. et al., 2004. Development \& validation of an organizational climate measure. , (0121).

Patterson, M.G. et al., 2005. Validating the organizational climate measure: links to managerial practices, productivity and innovation. Journal of Organizational Behavior, 408, pp.379-408.

Paullay, I.M., Alliger, G.M. \& Stone-Romero, E.F., 1994. Construct validation of two instruments designed to measure job involvement and work centrality. Journal of Applied Psychology, 79(2), pp.224-228.

Pugh, D.S. \& Hickson, D.J., 1996. Writers on Organizations: An invaluable introduction to the ideas and arguments of leading authorities on management 5th ed., Harmondsworth, Middlesex, England: Penguin Books.

Quinn, R.E., 1988. Beyond rational management: Mastering paradoxes and competing demands of high effectiveness, San Francisco, California: Jossey-Bass.

Quinn, R.E. \& Rohrbaugh, J., 1983. A Spatial Model of effectiveness criteria: towards a competing values approach to organizational analysis. Management Science, 29(3), pp.363-377.

Reino, A. \& Vadi, M., 2010. What factors predict the values of an organization and how? Organization, pp.1-45.

Renckly, T.R., 2002. Sampling and Surveying Handbook; Guidelines for planning, organizing, and conducting surveys 5th ed., USA: Air University.

Riggle, R.J., 2007. The impact of organizational climate variables of perceived organizational support, workplace isolation, and ethical climate on salesperson psychological and behavioral work outcomes. University of South Florida.

EJBE Vol. 4 No. 1/2014

Page 146 
Implications of Employees' Perception of Organizational Climate

Robbins, S.P., 2004. Organizational behavior 10th ed., New Jersey: PrenticeHall Inc.

Rose, N., 2005. Human Relations Theory and People Management. Human Relations, pp.43-62.

Saari, L.M. \& Judge, T.A., 2004. Employee Attitudes and Job Satisfaction. Human Resource Management, 43(4), pp.395-407.

Samuel, M.O. \& Chipunza, C., 2009. Employee retention and turnover : Using motivational variables as a panacea. African Journal of Business Management, 3(8), pp.410-415.

Schneider, B. \& Snyder, R.A., 1975. Some Relationships Between Job Satisfaction and Organizational Climate. Journal of Applied Psychology, 60(3), pp.318-328.

Speier, C. \& Venkatesh, V., 2002. The hidden minefields in the adoption of sales force automation technologies. Journal of Marketing, 66(3), pp.98111.

Srivastav, A.K., 2009. Heterogeneity of Organisational Climate. Research and Practice in Human Resource Management, 17(2), pp.1-13.

Steers, R.M., 1977. Antecedents and Outcomes of Organizational Commitment. Administrative Science Quarterly, 22, pp.46-56.

Veaux, D. et al., 2008. Stats Data and Models, New York: Pearson Education Inc.

Vijayakumar, V.S.R., 2007. Management Styles, Work Values and Organizational Climate. Journal of the Indian Academy of Applied Psychology, 33(2), pp.249-260.

Wachira, F.N., 2011. Public Sector Human Resource Managers Promoting Professionalism and Implementing the Public Service Charter at National 
Implications of Employees' Perception of Organizational Climate

Level: Roles, Facilitating and Inhibiting Factors and Strategic Actions. In

Discussion Paper. pp. 1-14.

Zafft, C.R., Adams, S.G. \& Matkin, G.S., 2009. Measuring Leadership in SelfManaged Teams Using the Competing Values Framework. Journal of Engineering Education, (July), pp.273-282. 